\title{
Hematoma intradiploico: tratamento cirúrgico e relato de caso
}

\author{
Ricardo de Amoreira Gepp ${ }^{1}$, Eidmar Augusto Neri', \\ Altamir Monteiro Junior ${ }^{1}$, Ricardo Karam Kalil ${ }^{1}$ \\ Rede Sarah de Hospitais de Reabilitação, Brasília, DF, Brasil.
}

\section{RESUMO}

O hematoma intradiploico é uma lesão rara do crânio. Foi descrita inicialmente em 1934 e até hoje a sua patogênese não é conhecida. Trata-se de uma lesão rara e secundária a um processo reativo benigno após traumas cranianos leves ou coagulopatias. Paciente de 42 anos apresentava quadro progressivo de perda da marcha e alteração visual. Foi identificada massa na região occipital do paciente, de caráter progressivo. Os estudos radiológicos demonstravam lesão de aspecto heterogêneo, erosiva da calota craniana e com expansão intracraniana. Realizou angiografia digital que demonstrou trombose de seio sagital superior. Foi submetido à biópsia seguida de ressecção da lesão associada à cranioplastia. Houve melhora do padrão visual e do desempenho de marcha do paciente, em relação ao pré-operatório. Os autores relatam um caso raro de hematoma intradiploico, analisaram os diagnósticos diferenciais e discutem as opções cirúrgicas dessa rara doença.

\section{PALAVRAS-CHAVE}

Hematoma epidural craniano/diagnóstico, hematoma epidural craniano/radiografia, hematoma epidural craniano/cirurgia, traumatismos craniocerebrais.

\section{ABSTRACT}

Intradiploic hematoma: surgical treatment and case report

Intradiploic hematoma is a very rare lesion of the skull. It was first described in 1934 and until now his pathogenesis still unclear. It is a rare and benign reactive process that occurs after minor head trauma. This 42-year-old man presented with difficult to walk and visual loss. It was observed one growing mass at occipital bone. On computadorized tomography and magnetic resonance image one destructive lesion, heterogeneous and expansion. One angiographic study was performed and showed thrombosis of the superior sagital sinus. The patient was submitted to a biopsy and after the hematoma was radically resected with cranioplasty. The improved his walk condition and visual capacities. The authors reported one case of intradiploic hematoma, differential diagnosis were analyzed and discussed about the surgical options of this rare disease.

\section{KEYWORDS}

Hematoma epidural cranial/diagnosis, hematoma epidural cranial/radiography, hematoma epidural cranial/surgery, craniocerebral trauma.

\section{Introdução}

O hematoma intradiploico é uma condição clínica rara, tendo sido descrito originalmente por Chorobski em 1934..$^{2,3}$ Os autores relatam um caso de um paciente com um grande hematoma intradiploico com extensão intra e extracraniana, que resultou em trombose da porção posterior do seio sagital superior. Existem poucos relatos na literatura sobre essa lesão expansiva de características não neoplásicas do crânio, que deve ser diferenciada de outras entidades clínicas. ${ }^{1,3}$ A maioria dos relatos realizados na literatura é de hematomas de pequeno volume e em pacientes pediátricos. Os autores descrevem um caso atípico de um hematoma intradiploico de grande volume em um paciente adulto e que ocasionou trombose de seio sagital superior, tendo sido o primeiro relato na literatura de estudo por angiografia digital de artérias carótidas e vertebrais, que demonstraram à trombose do seio secundário a compressão deste pelo hematoma crônico. 


\section{Relato do caso}

Paciente de 42 anos de idade, epiléptico desde os 7 anos, apresentou quadro de distúrbio progressivo para a marcha e alteração visual significativa. Aos 7 anos de idade, iniciou-se quadro de epilepsia, sendo a crise inicial caracterizada por uma crise parcial complexa com evolução para uma crise tônico-clônica generalizada, sendo inicialmente tratado com fenobarbital e fenitoína. Desde então, o paciente apresentou controle razoável das crises convulsivas.

O paciente gradativamente começou a apresentar um quadro de deterioração da memória e da atenção. Há cerca de cinco anos, notou-se deformidade em região occipital bilateral, pior do lado direito, deformidade essa caracterizada por tumoração de crescimento lento e progressivo. Há um ano, passou a evoluir com alguma dificuldade para manipular objetos, tendo deixado de se alimentar sozinho. Também há cerca de um ano, a mãe notou que o paciente não mais enxergava. Atualmente, o paciente apresenta grave dificuldade para a marcha, necessitando de auxílio ou uso de cadeira de rodas, assim como incontinência urinária, associadas a uma apatia severa. Apresenta-se com controle parcial das crises convulsivas.

Ao exame neurológico, apresentava-se consciente e apático. Observou-se deformidade na região parietoccipital, maior no lado direito, não dolorosa à palpação e não pulsátil. O perímetro cefálico era de $62 \mathrm{~cm}$. Apresentava quadro de cegueira cortical com componente anosognóstico. O fundo de olho estava normal, as pupilas eram de tamanho médio, fotorreativas. Trata-se de um paciente muito pouco orientado em relação ao tempo ou espaço e com dificuldade de aprendizado. Ele não conseguia lembrar-se de data, hora ou dia; exibia discreta apraxia manual; apresentava atrofia muscular, principalmente, com força muscular grau quatro nos membros. O paciente exibe uma posição distônica das mãos, com hipertonia. Os reflexos estavam globalmente diminuídos. Os sinais de Babinski e Hoffman foram negativos.

O paciente realizou estudo de tomografia computadorizada (TC) e ressonância magnética do encéfalo. Os exames de imagem demonstraram uma grande lesão intra e extracraniana. $\mathrm{Na}$ TC, observaram-se a presença de destruição óssea e sinais de que a lesão iniciou-se na calota (Figura 1). A RMN demonstrou ser uma lesão que não invade o encéfalo, com extensa área de reação do tecido subgaleal e que vai até a região da confluência dos seios venosos (Figura 2). Foram realizados exames laboratoriais, todos normais, não demonstrando alterações hematológicas, metabólicas ou infecciosas. Por causa da proximidade dos seios venosos, foi realizada uma angiografia digital, que demonstrou ausência de fluxo sanguíneo na região do tumor e uma trombose da porção distal do seio sagital superior (Figura 3).

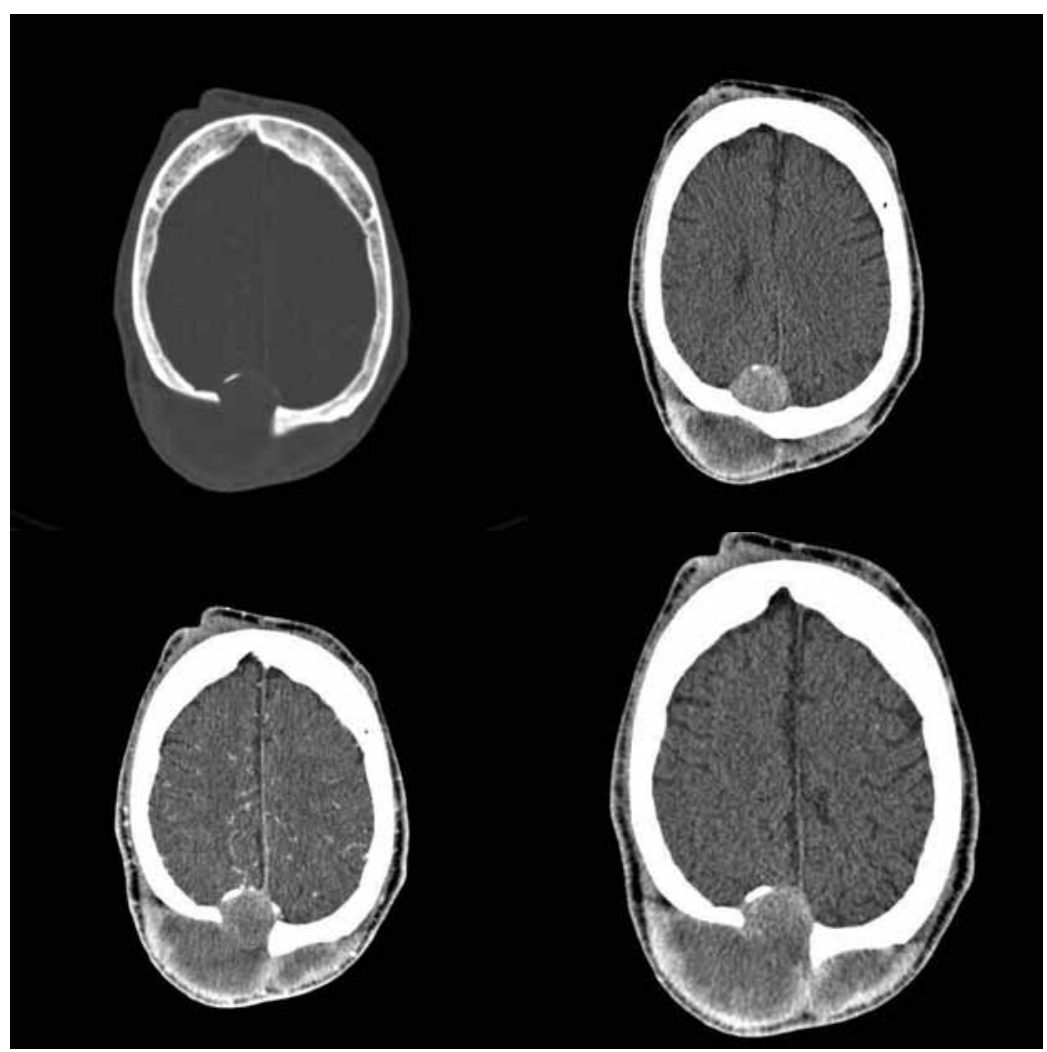

Figura 1 - Imagens de tomografia computadorizada demonstrando a erosão óssea e o componente intra e extracraniano do hematoma intradiploico. 


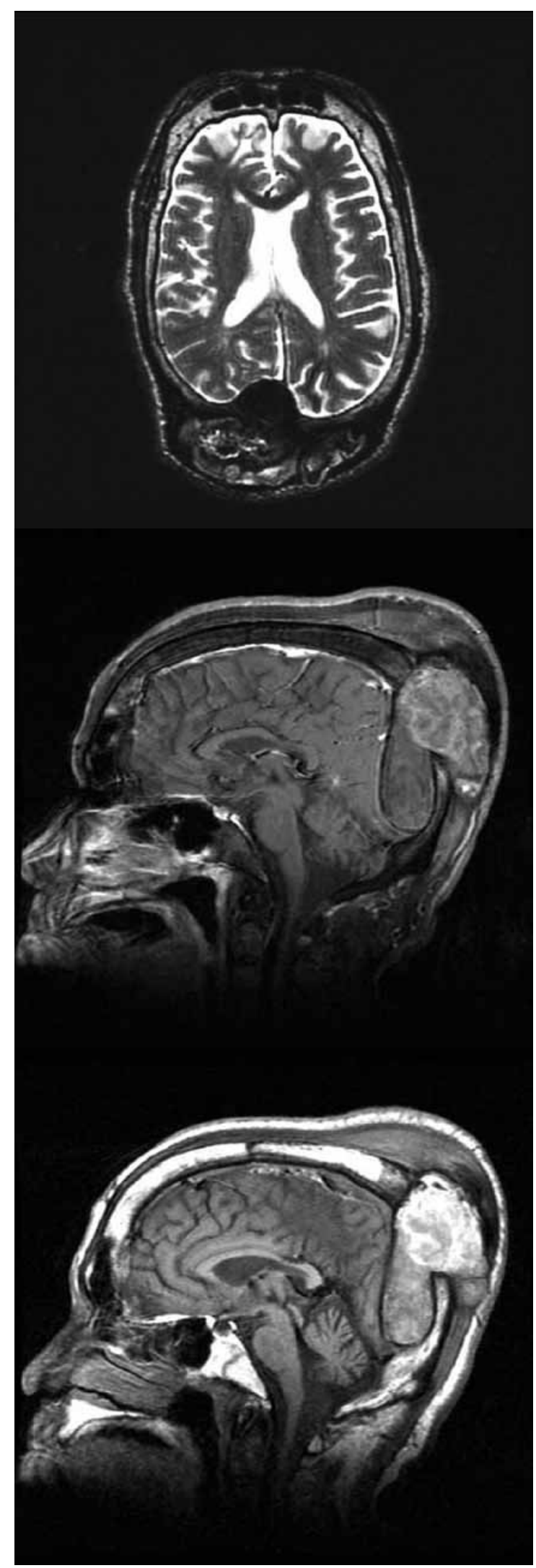

Figura 2 - Sequências de imagem de ressonância magnética demonstrando uma grande lesão expansiva intra e extracraniana. Observa-se a reação tecidual da gálea aponeurótica.

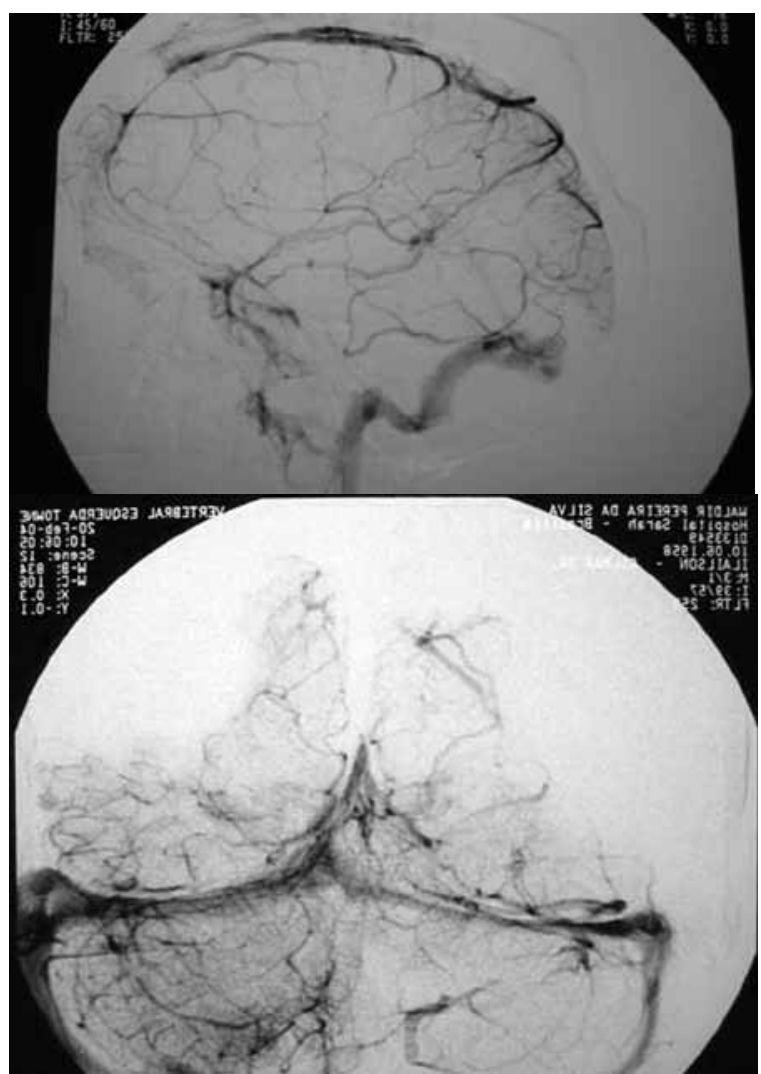

Figura 3 - Angiografia digital nas incidências de frente e perfil demonstrando a trombose do seio sagital superior secundário ao hematoma intradiploico.

Foi realizada uma excisão cirúrgica da lesão, sendo inicialmente encontrado um hematoma organizado e bastante aderido à gálea aponeurótica. Foi feita a retirada da lesão extracraniana em bloco, ficando a porção intracraniana. Foi realizada uma craniectomia ao redor do orifício no crânio e retirada da lesão intracraniana, que era completamente extradural. Após a regularização do osso, foi realizada uma cranioplastia com acrílico. Realizou-se exame de controle, que demonstrou ressecção adequada da lesão e adequado posicionamento da cranioplastia (Figura 4).

O diagnóstico foi de um hematoma intradiploico crônico. A tumoração extracraniana tinha 258 g, com $11 \times 10 \times 4 \mathrm{~cm}$ de dimensões. A lesão caracterizava-se por uma lesão reacional com capa de tecido fibroso periférica, densa, com hemossiderófagos, células gigantes multinucleadas, fendas de colesterol infiltrado linfocítico e metaplasia óssea focal. Conteúdo hemorragia, fibrina e tecido degenerado.

O paciente evoluiu de forma adequada no período pós-operatório. Houve melhora da capacidade visual após seis meses do procedimento cirúrgico. Após período de quatro anos de pós-operatório, o paciente apresentava controle das crises convulsivas e houve melhora da visão. 


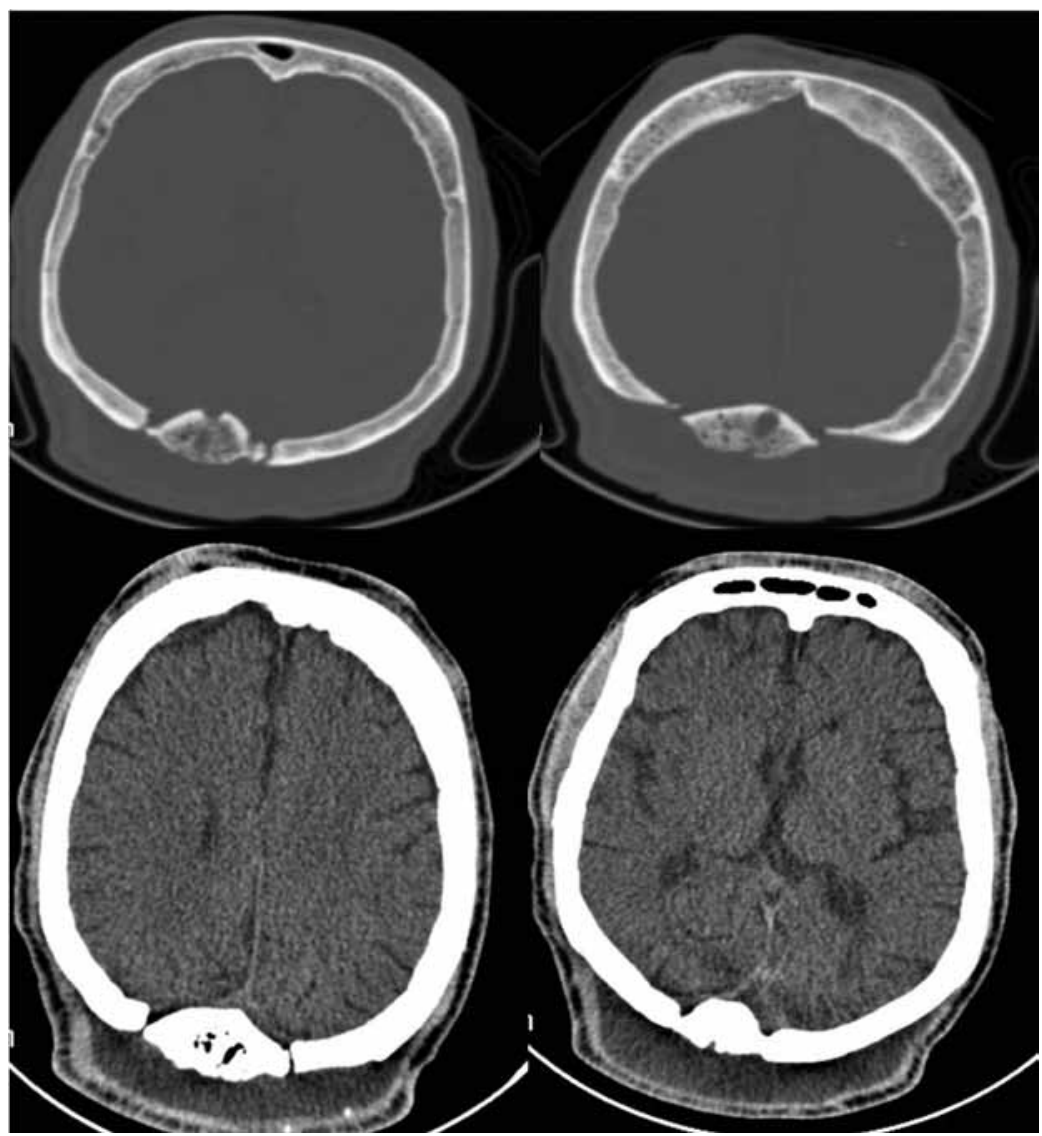

Figura 4 - Tomografia de controle pós-operatório. Os exames na parte superior demonstram a cranioplastia realizada com acrílico. As imagens na porção inferior demonstram a resolução do hematoma.

\section{Discussão}

O hematoma intradiploico é uma entidade clínica rara. ${ }^{1,3}$ Em 1934, Chorobski e Davis descreveram um cisto intradiploico que foi diagnosticado anos após um trauma craniano. ${ }^{3,6} \mathrm{~A}$ formação dessa lesão está relacionada a pequenos traumas cranianos em adultos, e em recém-nascidos encontrou-se a deficiência do fator VIII como um fator de predisposição para o surgimento da lesão. ${ }^{1}$ Nos casos descritos até o momento, com exceção de um onde houve um trauma grave, todos os casos ocorreram após pequenos traumas cranianos e que ocorreram de forma repetitiva ${ }^{1,2,5}$ (Tabela 1).

O diagnóstico do hematoma intradiploico crônico é feito pela imagem pré-operatória e confirmado pela análise histopatológica. A lesão apresenta um comportamento progressivo, com erosão da calota craniana e formação de um tecido reacional fibrótico importante e associado a um tecido osteoide. ${ }^{2,8}$ Observa-se também a presença de hematoma antigo encapsulado por um cisto em que as paredes são compostas de tecido de granulação ou colágeno. ${ }^{1,5}$

Os diagnósticos diferenciais são importantes diante de uma imagem de uma lesão expansiva do crânio.
O diagnóstico de hematoma intradiploico é uma exceção e lesões como: cisto epidermoide intradiploico, meningioma intraósseo, hemangiomas ósseos, cisto de aracnoide intradiploico, lesões metastáticas e doenças granulomatosas. ${ }^{4,5,7}$

Os relatos existentes na literatura descrevem lesões de pequenas dimensões na calota craniana. A maioria dos relatos descreve hematomas em recém-nascidos e associados a doenças hematológicas, sendo o fato da ocorrência de distúrbios da coagulação um fator comum. ${ }^{2,5}$ Apesar de ser uma doença benigna, ela tem um comportamento progressivo e, como demonstrado neste caso, pode ocasionar déficit neurológico grave e alterações secundárias importantes. ${ }^{2}$

$\mathrm{O}$ tratamento dessa lesão é cirúrgico. A retirada completa da lesão seguida de curetagem de todo o tecido de preenchimento é o tratamento de escolha e deve ser seguido de hemostasia das bordas ósseas. ${ }^{3,6}$ Ocasionalmente, a biópsia por agulha guiada por imagem pode preceder a ressecção da lesão, permitindo melhor planejamento cirúrgico já com o diagnóstico histológico da lesão. Após a ressecção completa do hematoma, deve ser realizada a cranioplastia nas lesões amplas e que erodiram também a tábua óssea interna. A cranioplastia pode 


\begin{tabular}{|c|c|c|c|c|}
\hline \multicolumn{5}{|c|}{$\begin{array}{c}\text { Tabela } 1 \text { - A tabela acima descreve resumidamente } \\
\text { as referências atuais encontradas na literatura e } \\
\text { a forma de tratamento cirúrgico realizada }\end{array}$} \\
\hline Autor & Osso & Exame & Patologia & Tratamento \\
\hline $\begin{array}{l}\text { Marcado, } \\
1984\end{array}$ & Parietal & TC & $\begin{array}{l}\text { Granuloma } \\
\text { reparativo }\end{array}$ & $\begin{array}{l}\text { Excisão e } \\
\text { cranioplastia }\end{array}$ \\
\hline $\begin{array}{l}\text { Palantinsky, } \\
1986\end{array}$ & Parietal & $\mathrm{TC}$ & $\begin{array}{l}\text { Granuloma e } \\
\text { hematoma }\end{array}$ & $\begin{array}{l}\text { Excisão e } \\
\text { cranioplastia }\end{array}$ \\
\hline Yuasa, 1992 & Parietal & TC & $\begin{array}{l}\text { Hematoma } \\
\text { diploico }\end{array}$ & $\begin{array}{l}\text { Excisão e } \\
\text { cranioplastia }\end{array}$ \\
\hline Sato, 1994 & Parietal & $\mathrm{RMN}$ & $\begin{array}{l}\text { Hematoma } \\
\text { intradiploico }\end{array}$ & $\begin{array}{l}\text { Excisão e } \\
\text { cranioplastia }\end{array}$ \\
\hline Goel, 1996 & Frontal & TC & $\begin{array}{l}\text { Granuloma } \\
\text { hematoma }\end{array}$ & $\begin{array}{l}\text { Evacuação } \\
\text { do } \\
\text { hematoma }\end{array}$ \\
\hline $\begin{array}{l}\text { Uemura, } \\
1997\end{array}$ & Frontal & $\mathrm{RMN}$ & $\begin{array}{l}\text { Hematoma } \\
\text { intraósseo }\end{array}$ & Excisão \\
\hline $\begin{array}{l}\text { Yucesoy, } \\
1999\end{array}$ & Parietal & TC & $\begin{array}{l}\text { Hematoma } \\
\text { organizado }\end{array}$ & Excisão \\
\hline $\begin{array}{l}\text { Mobbs, } \\
2000\end{array}$ & Parietal & $\mathrm{RMN} / \mathrm{TC}$ & $\begin{array}{l}\text { Hematoma } \\
\text { organizado }\end{array}$ & Curetagem \\
\hline Nagis, 2005 & Frontal & TC & $\begin{array}{l}\text { Hematoma } \\
\text { organizado }\end{array}$ & Excisão \\
\hline Dange, 2010 & Frontal & $\mathrm{RMN}$ & $\begin{array}{l}\text { Hematoma } \\
\text { organizado }\end{array}$ & Excisão \\
\hline Relato atual & Occipital & $\begin{array}{l}\mathrm{RMN} / \mathrm{TC} \\
\text { e angio }\end{array}$ & $\begin{array}{l}\text { Hematoma } \\
\text { organizado }\end{array}$ & $\begin{array}{l}\text { Excisão e } \\
\text { cranioplastia }\end{array}$ \\
\hline
\end{tabular}

ser feita com osso retirado da própria calota craniana ou preferencialmente utilizando material sintético como acrílico, evitando assim uma área cruenta no osso que possa gerar novo hematoma.

O hematoma intradiploico é uma doença rara, de características benignas, mas que, pelo comportamento progressivo, pode ocasionar graves alterações neurológicas no paciente. ${ }^{2} \mathrm{O}$ neurocirurgião deve estar atento a essa possibilidade diagnóstica diante de uma lesão expansiva do crânio em pacientes submetidos a trauma repetitivo ou distúrbios da coagulação.

\section{Referências}

1. Dange N, Mahore A, Avinash KM, Joshi V, Kawale J, Goel A. Chronic intradiploic hematoma in patients with coagulopathy. J Clin Neurosci. 2010;17(8):1047-9.

2. Goel A, Nadkarni T, Mazumdar D. Intradiploic haematoma behaving as a progressive mass lesion. $\mathrm{Br} \mathrm{J}$ Neurosurg. 1996;10(6):611-2.

3. Mobbs RJ, Gollapudi PR, Fuller JW, Dahlstrom JE, Chandran NK. Intradiploic hematoma after skull fracture: case report and literature review. Surg Neurol. 2000;54(1):87-91.

4. Nagi S, Megdiche H, Daoud H, Bouchriha M, Sebaï R, Belghith L, et al. [Intradiploic hematoma: report of two cases and review of literature]. Tunis Med. 2005;83(7):426-8.

5. Reeves A, Edwards-Brown M. Intraosseous hematoma in a newborn with factor VIII deficiency. AJNR Am J Neuroradiol. 2000;21(2):308-9.

6. Sato K, Kubota T, Kawano H. Chronic diploic hematoma of the parietal bone. Case report. J Neurosurg. 1994;80(6):1112-5.

7. Wani AA, Ramzan AU, Kirmani AR, Bhatt AR, Hamdani N, Zargar J. Intradiploic epidermoid causing spontaneous extradural hematoma: case report. Neurosurgery. 2008;62(4):E971.

8. Yuasa $\mathrm{H}$, Watanabe $\mathrm{H}$, Uemura $\mathrm{Y}$, Tokito $\mathrm{S}$. Intraosseous hematoma of the skull: case report. Neurosurgery. 1992;30(5):776-8.

Endereço para correspondência

Ricardo de Amoreira Gepp

SQSW, 300, bloco M, ap. 204, Sudoeste

70673-014 - Brasília, Distrito Federal, Brazil

Telefone: (61) 3342-2420

E-mails: ricardogepp@zipmail.com.br; rgepp@sarah.br 